\title{
Soroprevalência da infecção chagásica no Estado do Piauí, 2002
}

\author{
Seroprevalence of Chagas disease infection \\ in the State of Piauí, 2002
}

\author{
José Borges-Pereira ${ }^{1}$, José Adail Fonseca de Castro ${ }^{3}$, Arlete Gonçalves da Silva ${ }^{4}$, Patrícia Lago \\ Zauza $^{1}$, Tiago Pires Bulhões ${ }^{1}$, Maria Elizabete Gonçalves ${ }^{2}$, Ernani Saraiva de Almeida ${ }^{2}$, \\ Maria do Amparo Salmito², Lucia Regina Montebello Pereira ${ }^{2}$, Francisco Itamar Alves Filho ${ }^{3}$, \\ Fernando G. Correia-Lima ${ }^{2}$ e José Rodrigues Coura ${ }^{1}$
}

\begin{abstract}
RESUMO
Com o objetivo de avaliar a situação epidemiológica da infecção chagásica no Estado do Piauí e sua relação com idade, gênero, transfusão de sangue e aborto espontâneo, foi realizado, de agosto a dezembro de 2002, um inquérito sorológico com o exame de uma amostra aleatória simples de 36.399 moradores da área rural. A infecção chagásica foi definida pelo teste de imunofluorescência indireta para anticorpos antiTrypanosoma cruzi em amostra de sangue coletada em papel de filtro. A soroprevalência total foi de 1,9\%, variando de 0,1\% em menores de 5 anos a 6,6\% em maiores de 79 anos; foi significativamente maior nas mulheres $(2,1 \%)$, analfabetos $(4,1 \%)$, receptores de sangue $(3,3 \%)$ e nas mulheres com abortamento espontâneo (5,4\%). Esses dados ao serem comparados com os obtidos durante o inquérito sorológico nacional (1975-1980) mostraram significativa queda da soroprevalência da infecção chagásica no Estado do Piauí (4\% para 1,9\%), indicando a eficácia das medidas de controle vetorial implementadas no período 1975-2002.
\end{abstract}

Palavras-chaves: Vigilância epidemiológica. Soroprevalência da infecção chagásica. Transfusão de sangue. Aborto espontâneo. Estado do Piauí.

\begin{abstract}
To evaluate the epidemiological situation of Chagas disease infection in the State of Piauí, Brazil, and its relationships with age, gender, blood transfusion and spontaneous abortion, a serological survey was performed. A random sample of 36,399 inhabitants of rural zones was examined between August and December 2002. Chagas disease infection was defined by the indirect immunofluorescence test, with identification of antiTrypanosoma cruzi antibodies in blood samples collected on filter paper. The total seroprevalence was $1.9 \%$, ranging from $0.1 \%$ in children less than five years old to $6.6 \%$ in adults over 79 years old. The seroprevalence was significantly higher in women (2.1\%), illiterates (4.1\%), individuals receiving blood (3.3\%) and women with a history of spontaneous abortion (5.4\%). Comparison with the national serological survey (1975-1980) showed that there had been a significant reduction in the seroprevalence of Chagas disease infection in the State of Piauí (4.0\% to 1.9\%), which was indicative of the efficacy of the vector control measures implemented between 1975 and 2002.
\end{abstract}

Key-words: Epidemiological surveillance. Seroprevalence of Chagas disease infection. Blood transfusion. Spontaneous abortion. State of Piauí.

\footnotetext{
1.Departamento de Medicina Tropical do Instituto Oswaldo Cruz, Fundação Oswaldo Cruz, Rio de Janeiro, RJ. 2. Secretaria de Saúde do Estado do Piauí, Teresina, PI. 3. Departamento de Microbiologia e Parasitologia da Universidade Federal do Piaú́, Teresina, PI. 4. Departamento de Parasitologia da Universidade do Estado do Piauí, Teresina, PI.

Apoio da Secretaria de Saúde do Estado do Piauí, Universidade Federal do Piauí e Fundação Oswaldo Cruz

Endereço para correspondência: Dr. José Borges-Pereira. Laboratório de Doenças Parasitárias/Depto. Medicina Tropical/FIOCRUZ. Av. Brasil, 4365, Pav. Arthur Neiva, sala 17, Manguinhos, 21045-900 Rio de Janeiro, RJ.

e-mail: borges@ioc.fiocruz.br

Recebido para publicação em 27/1/2005

Aceito em 10/10/2006
} 
A história da doença de Chagas no Estado do Piauí iniciase em 1916, com os relatos de Neiva \& Penna ${ }^{15}$ mencionando a presença de indivíduos com queixas de entalo e vexame, sugestivas de megaesôfago e cardiopatia e a captura de Triatoma brasiliensis, Triatoma maculata, Triatoma sordida e Panstrongylus megistus nos municípios de São Raimundo Nonato, Parnaguá e Corrente, com alguns exemplares infectados por Trypanosoma cruzi. Em 1975, Figueiredo e cols ${ }^{12}$ confirmaram os primeiros casos autóctones da doença de Chagas com manifestações cardíacas e digestivas provenientes de Oeiras, Castelo e Bom Jesus do Gurguéia.

A partir de 1975, a soroprevalência da infecção chagásica foi abordada em estudos pontuais realizados em áreas de Oeiras ${ }^{911}$, Castelo do Piauí, Pedro II $^{3}$ e João Costa ${ }^{6}$. A primeira abordagem de toda a população foi feita através do inquérito sorológico nacional ${ }^{7}$ envolvendo moradores da área rural dos 114 municípios do Estado do Piauí, desenvolvido no período 1975/1977. Nesse estudo, por amostragem, estimou-se em $4 \%$ a soroprevalência da infecção chagásica para a população do Estado, com índices de soropositividade em todas as faixas etárias e indicações de atividade da transmissão vetorial. No período de 1996/1997 foi realizado um outro inquérito sorológico envolvendo 4.989 escolares de 7 a 14 anos procedentes de 14 municípios, no qual foram registrados dois $(0,04 \%)$ casos soropositivos ${ }^{17}$ e indicações de expressiva redução da transmissão da infecção. Esses resultados mostraram a tendência de queda da prevalência da infecção chagásica humana em alguns municípios do Estado do Piauí, mas, não permitiram a avaliação da eficácia das medidas de controle de todos os municípios do Estado, na perspectiva das atribuições preconizadas pelo Sistema Único de Saúde (SUS).

Produzir resultados que permitam a cada administração municipal monitorar os mecanismos de transmissão da infecção chagásica através da soroprevalência é o principal objetivo desse estudo envolvendo amostras da população rural de todos os municípios existentes no Estado do Piauí, até dezembro de 2002.

\section{MATERIAL E MÉTODOS}

Área e população de estudo. 0 Estado do Piauí localizase entre $2^{0} 44^{\prime} 49^{\prime \prime}$ e $10^{\circ} 55^{\prime} 05^{\prime}$ de latitude sul e entre $40^{\circ} 22^{\prime} 12^{\prime \prime}$ e 45 59 ' de longitude oeste. Apresenta clima tipicamente tropical com temperaturas médias elevadas, variando entre $18^{\circ} \mathrm{C}$ e $39^{\circ} \mathrm{C}$ e umidade relativa do ar oscilando de 60 a $86 \%$. Ocupa uma área de $251.529 \mathrm{~km}^{2}$, dividida em 222 municípios e limitada pelos Estados do Maranhão, Tocantins, Bahia, Pernambuco e Ceará e pelo Oceano Atlântico (Figura 1). De acordo com o Censo $2000^{13}$, apresentava uma população de 2.843 .689 pessoas, sendo $50,8 \%$ do sexo feminino e $49,2 \%$ do sexo masculino; 1.788 .179 (62,9\%) moradores da área urbana e 1.055 .510 (37,1\%) da área rural (distribuída por faixa etária na Tabela 1). Os 222 municípios estão agrupados em 17 Regionais de Saúde ou Distritos Sanitários (Tabela 2).
Tabela 1 - População rural e examinada no inquérito sorológico para doença de Chagas, de acordo com a faixa etária, Piauí, 2002.

\begin{tabular}{lrrrrr}
\hline & \multicolumn{2}{c}{ População rural* } & & \multicolumn{2}{c}{ População examinada } \\
\cline { 2 - 3 } Faixa etária(anos) & \multicolumn{1}{c}{$\mathrm{n}^{0}$} & $\%$ & & $\mathrm{n}^{0}$ & $\%$ \\
\hline $00-04$ & 123.920 & 11,7 & 3.888 & 10,7 \\
$05-09$ & 125.334 & 11,9 & 4.540 & 12,5 \\
$10-14$ & 139.797 & 13,3 & 4.390 & 12,1 \\
$15-19$ & 128.689 & 12,2 & 3.879 & 10,7 \\
$20-29$ & 163.941 & 15,5 & 5.471 & 15,1 \\
$30-39$ & 118.365 & 11,2 & 4.155 & 11,5 \\
$40-49$ & 93.600 & 8,9 & 3.292 & 9,1 \\
$50-59$ & 72.566 & 6,9 & 2.854 & 7,9 \\
$60-69$ & 50.599 & 4,8 & 2.045 & 5,6 \\
$70-79$ & 26.968 & 2,6 & 1.158 & 3,2 \\
$\geq 80$ & 11.320 & 1,1 & 562 & 1,6 \\
Ignorada & 411 & 0,1 & 165 & 0,5 \\
\hline Total & 1.055 .510 & 100,0 & 36.399 & 100,0 \\
\hline
\end{tabular}

*Censo demográfico 2000 - IBGE

Tabela 2 - Soropositividade para anticorpos antiTrypanosoma cruzi em moradores da área rural do Estado do Piauí, 2002, de acordo com a Regional de Saúde.

\begin{tabular}{|c|c|c|c|c|}
\hline \multirow[b]{2}{*}{ № } & \multirow{2}{*}{$\begin{array}{l}\text { Regional de Saúde } \\
\text { Município sede }\end{array}$} & \multicolumn{3}{|c|}{ População rural } \\
\hline & & examinada & soropos* & soropos (\%) \\
\hline $1^{\mathrm{a}}$ & Parnaíba & 2.467 & 19 & 0,8 \\
\hline $2^{\mathrm{a}}$ & Barras & 3.225 & 23 & 0,7 \\
\hline $3^{\mathrm{a}}$ & Piripiri & 2.319 & 10 & 0,4 \\
\hline $4^{\mathrm{a}}$ & Teresina & 3.894 & 17 & 0,4 \\
\hline $5^{\mathrm{a}}$ & Campo Maior & 2.285 & 25 & 1,1 \\
\hline $6^{\mathrm{a}}$ & Amarante & 1.462 & 5 & 0,3 \\
\hline $7 \underline{a}$ & Valença & 1.441 & 16 & 1,1 \\
\hline $8^{\mathrm{a}}$ & Oeiras & 1.888 & 110 & 5,8 \\
\hline $9^{\mathrm{a}}$ & Picos & 2.932 & 127 & 4,3 \\
\hline $10^{\mathrm{a}}$ & Floriano & 2.289 & 43 & 1,9 \\
\hline $11^{\mathrm{a}}$ & São João do Piauí & 2.399 & 126 & 5,3 \\
\hline $12^{\mathrm{a}}$ & São Raimundo Nonato & 1.890 & 34 & 1,8 \\
\hline $13^{\mathrm{a}}$ & Bom Jesus & 1.324 & 17 & 1,3 \\
\hline $14^{\mathrm{a}}$ & Corrente & 2.434 & 98 & 4,0 \\
\hline $15^{\mathrm{a}}$ & Uruçuí & 560 & 10 & 1,8 \\
\hline $16^{\mathrm{a}}$ & Fronteira & 1.250 & 2 & 0,2 \\
\hline $17^{\mathrm{a}}$ & Paulistana & 2.340 & 14 & 0,6 \\
\hline Total & & 36.399 & 696 & 1,9 \\
\hline
\end{tabular}

* Soropositivos pelo teste de imunofluorescência indireta em eluato de sangue

Amostragem. 0 alvo do inquérito foi a população da área rural dos 222 municípios do Estado, de ambos os sexos e todas as idades. Para atender a capacidade operacional dos órgãos executores, demandas municipais acerca do atendimento à população chagásica e planejamento de estratégias de controle da transmissão vetorial, definiu-se uma amostra mínima de 5\% da população rural. 0 número de domicílios a ser visitado foi calculado dividindo o total estimado de participantes pela média de 4 habitantes por domicílio do estado, estabelecida no censo demográfico $2000^{13}$. Para definir quais os domicílios seriam visitados em cada município, utilizamos o cadastro das localidades do programa de saúde da família (PSF) e sorteamos as localidades rurais com 20 ou mais domicílios, empregando a tabela de números aleatórios, até atingir o número estimado. Com essa metodologia, foi estabelecida, para todo o estado, 


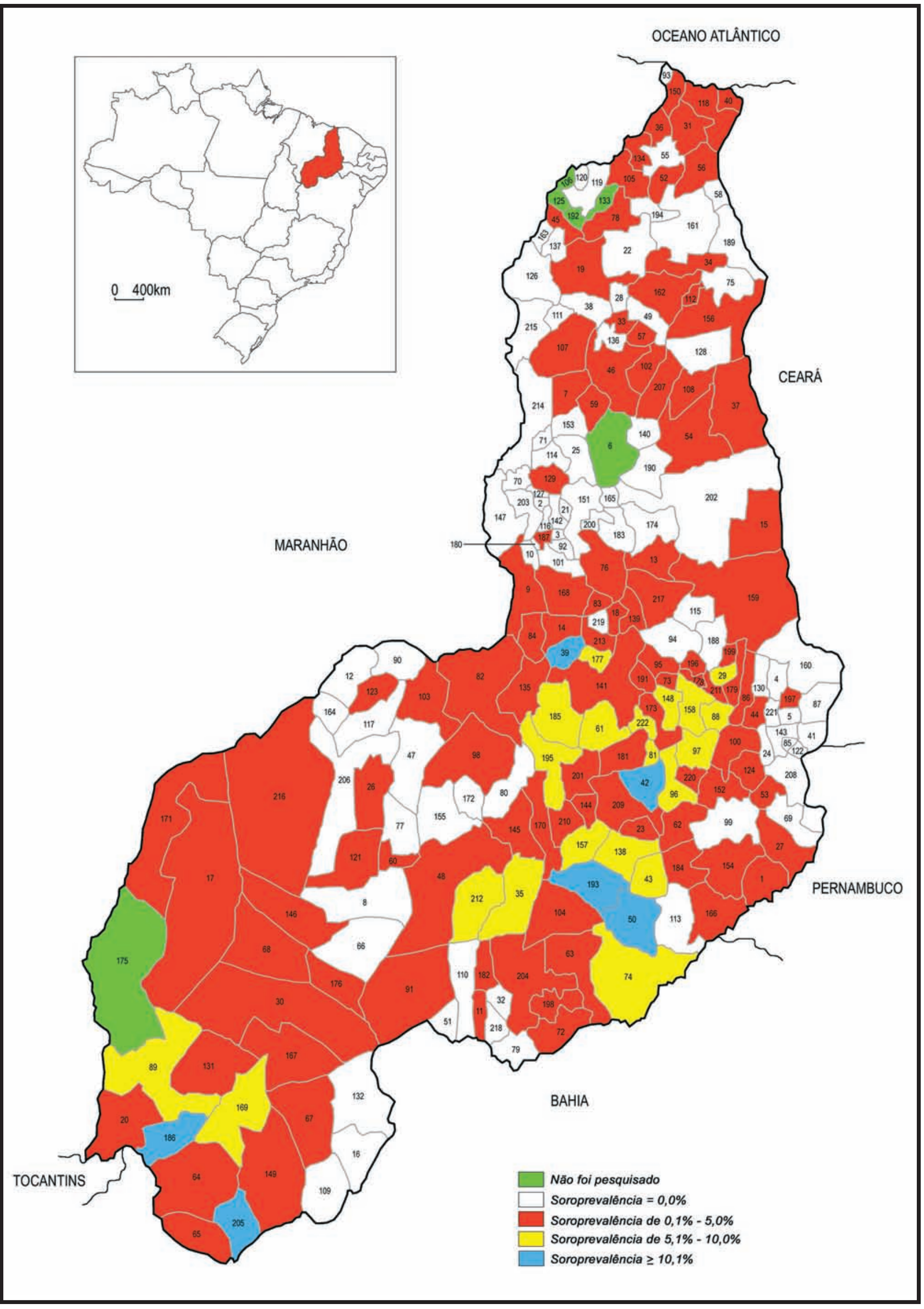

Figura 1 - Distribuição da soroprevalência para doença de Chagas no Estado do Piauí, 2002. 
uma amostra de 53.810 moradores de 12.700 domicílios, a qual teria sido 10 vezes menor se a demanda fosse exclusivamente da administração estadual.

Triagem sorológica. No período de agosto a dezembro de 2002, foi realizada a coleta de sangue para a pesquisa de anticorpos antiTrypanosoma cruzi com a técnica qualitativa de imunofluorescência indireta (IFI) preconizada por Souza e Camargo ${ }^{18}$. Após as informações sobre a pesquisa e autorização obtida das pessoas presentes nos domicílios, no momento da visita dos agentes de saúde, foram coletadas amostras de sangue da polpa digital em papel de filtro (Klabin80, fornecido pela Secretaria de Saúde do Estado do Piauí), as quais foram mantidas em meio ambiente, no máximo, por 10 dias e armazenadas em geladeira com temperaturas de 2 a $8^{\circ} \mathrm{C}$, no máximo, por 60 dias, até a realização dos testes. No processamento das amostras de sangue, para a eluição, aos discos de 1,2mm de diâmetro depositados em pequenos botoques de plástico, adicionava-se $0,2 \mathrm{ml}$ de solução salina tamponada com fosfatos de $0,01 \mathrm{M}, \mathrm{pH} 7,2$ e deixava pelo tempo mínimo de 3 horas. Em seguida, 1 gota do eluato, cerca de 20 microlitros, era transferida para a área demarcada da lâmina na qual estavam fixadas as formas epimastigotas do T. cruzi empregadas como antígenos.

No total, foram coletadas amostras de sangue de 36.399 pessoas residentes em 11.439 domicílios de 216 municípios. As amostras de sangue foram submetidas ao teste de IFI (Kit lote 024CI003Z, produzido por Bio-Manguinhos) fornecido aos Departamentos de Microbiologia e Parasitologia da Universidade Federal do Piauí, Teresina, PI e de Medicina Tropical do Instituto Oswaldo Cruz, Rio de Janeiro. 0 controle de qualidade das amostras positivas e de igual número de amostras negativas foi realizado no setor de sorologia do Laboratório de Doenças Parasitárias do Departamento de Medicina Tropical do Instituto Oswaldo Cruz da Fundação Oswaldo Cruz, Rio de Janeiro, RJ, utilizando-se o mesmo lote do Kit produzido em Bio-Manguinhos. A soropositividade da infecção chagásica foi considerada a partir da confirmação do resultado positivo da amostra de sangue por dois observadores de cada laboratório.

Durante as visitas aos domicílios, foram aplicados questionários abordando questões relacionadas com a escolaridade na população com $\geq 10$ anos de idade, transfusão de sangue em toda a população e abortamento espontâneo nas mulheres com $\geq 15$ anos.

Análise estatística. Foram realizadas análises descritivas das variáveis com suas respectivas frequiências. As diferenças de freqüências foram avaliadas pelo teste quiquadrado, considerando-se o $\mathrm{p} \leq 0,05$ como significância estatística. A análise dos dados foi realizada através do programa EPI-INF0.6.

Aspectos éticos. 0 estudo foi aprovado pelo Comitê de Ética em Pesquisa da Universidade Federal do Piauí. As pessoas adultas e responsáveis por menores assinaram o Termo de Consentimento Informado Livre e Esclarecido, conforme a Resolução 196/1996 do Conselho Nacional de Saúde.

\section{RESULTADOS}

Desempenho. Do total de 222 municípios existentes, em $6(2,7 \%)$ não foi realizada a pesquisa planejada: Joca Marques, Matias Olímpio, São João do Arraial, Morro do Chapéu do Piauí, Alto Longá, e Santa Filomena pela ausência de pessoal no período destinado à pesquisa.

Do total de 12.700 domicílios estimados para a pesquisa, 11.439 (90\%) participaram da pesquisa através dos moradores e 1.261 (10\%) não participaram: 328 (2,6\%) dos seis municípios que não realizaram a pesquisa e 933 (7,3\%) estavam fechados ou não tinham moradores no momento da visita.

Dos 53.810 habitantes estimados para o exame, 36.399 (67,6\%) participaram, permitindo a coleta de amostra de sangue em papel de filtro e $17.411(32,4 \%)$ não participaram: $1.314(2,4 \%)$ moradores dos seis municípios que não realizaram a pesquisa, $4.412(8,2 \%)$ recusaram a coleta do sangue e 11.685 (21,7\%) não estavam no domicílio no momento da visita dos agentes de saúde. A distribuição, por faixa etária, das populações rural e examinada está assinalada na Tabela 1.

Soropositividade. Em todas as Regionais de Saúde, foram identificadas pessoas soropositivas para anticorpos antiT. cruzi, com prevalências variando de $0,2 \%$ a 5,8\% (Tabela 2). As Regionais de Oeiras, Picos, São João do Piauí e de Corrente apresentaram soropositividades acima da média, ressaltando-se que nessas áreas, em 1975, se concentravam os 15 municípios com captura positiva de T. infestans.

Dos 216 municípios pesquisados, em 131 (60,6\%) houve registro de pessoas soropositivas para anticorpos antiT. cruzi (Tabela 3), enquanto no inquérito sorológico nacional realizado no período 1975/1977, dos 114 municípios existentes e pesquisados, 103 (90,4\%) apresentaram pessoas soropositivas. 0 confronto desses resultados revela uma queda significativa no percentual de municípios com pessoas soropositivas, assim como no percentual de municípios com soroprevalência acima de 10\% (Tabela 4), indicando uma redução do espaço ocupado pela doença de Chagas.

Do total de 11.439 domicílios pesquisados, em 627 $(5,5 \%)$ foram identificadas pessoas soropositivas para anticorpos antiT. cruzi. Desses 627 domicílios, 567 (90,4\%) tinham uma pessoa soropositiva, $52(8,3 \%)$ tinham duas pessoas soropositivas, $7(1,1 \%)$ tinham três pessoas e $1(0,2 \%)$ tinha 4 pessoas.

Do total de 36.399 pessoas examinadas no presente inquérito, 696 (1,9\%) apresentaram o teste de IFI positivo para anticorpos antiT. cruzi. A soropositividade foi identificada em $401(2,1 \%)$ das 19.047 mulheres examinadas e em $295(1,7 \%)$ dos 17.352 homens examinados $\left(\chi^{2}=8,31 ; \mathrm{p}=0,0039\right.$; $\mathrm{gl}=1 ; \mathrm{RR}=1,24[1,07<\mathrm{RR}<1,44]) ;$ a soropositividade aumentou com a idade; variando de $0,1 \%$ entre os menores de 5 anos a 6,6\% nos maiores de 79 anos (Tabela 5). Destaca-se a idade média de 54,5 $\pm 17,2$ anos do grupo de pessoas soropositivas, significativamente maior do que a idade média de 27,4 $\pm 21,1$ anos do grupo de pessoas soronegativas. 
Tabela 3 - Soropositividade para anticorpos antiTrypanosoma cruzi em moradores da área rural do Estado do Piauí, 2002, de acordo com o município.

\begin{tabular}{|c|c|c|c|c|c|c|c|c|c|}
\hline \multirow{2}{*}{$\begin{array}{l}\text { №na } \\
\text { Figura } 1\end{array}$} & \multirow[b]{2}{*}{ Municípios } & \multicolumn{3}{|c|}{ Exames realizados } & \multirow{2}{*}{$\begin{array}{l}\text { №na } \\
\text { Figura } 1\end{array}$} & \multirow[b]{2}{*}{ Municípios } & \multicolumn{3}{|c|}{ Exames realizados } \\
\hline & & $\mathrm{n}^{0}$ & pos & $\%$ pos & & & $\overline{\mathrm{n}^{0}}$ & pos & $\%$ pos \\
\hline 1 & Acauã & 108 & 1 & 0,9 & 57 & Cocal de Telha & 82 & 1 & 1,2 \\
\hline 2 & Agricolândia & 78 & 0 & 0 & 58 & Cocal dos Alves & 149 & 0 & 2, \\
\hline 3 & Água Branca & 61 & 0 & 0 & 59 & Coivaras & 106 & 3 & 2,8 \\
\hline 4 & Alagoinha do PI & 62 & 0 & 0 & 60 & Colônia do Gurguéia & 65 & 1 & 1,5 \\
\hline 5 & Alegrete do PI & 35 & 0 & 0 & 61 & Colônia do PI & 134 & 7 & 5,2 \\
\hline 6 & Alto Longa & 0 & 0 & 0 & 62 & Conceição do Canindé & 63 & 2 & 3,2 \\
\hline 7 & Altos & 509 & 5 & 1,0 & 63 & Coronel José Dias & 100 & 4 & 4,0 \\
\hline 8 & Alvorada do Gurguéia & 190 & 0 & 0 & 64 & Corrente & 433 & 20 & 4,6 \\
\hline 9 & Amarante & 224 & 1 & 0,5 & 65 & Cristalândia do PI & 237 & 7 & 3,0 \\
\hline 10 & Angical do PI & 66 & 0 & 0 & 66 & Cristino Castro & 194 & 0 & 0 \\
\hline 11 & Anísio de Abreu & 149 & 1 & 0,7 & 67 & Curimatá & 132 & 4 & 3,0 \\
\hline 12 & Antonio Almeida & 38 & 0 & 0 & 68 & Currais & 117 & 1 & 0,9 \\
\hline 13 & Aroazes & 109 & 5 & 4,6 & 69 & Curral Novo do PI & 76 & 0 & 0 \\
\hline 14 & Arraial & 85 & 2 & 2,4 & 70 & Curralinhos & 137 & 0 & 0 \\
\hline 15 & Assunção do PI & 136 & 1 & 0,7 & 71 & Dermeval Lobão & 72 & 0 & 0 \\
\hline 16 & Avelino Lopes & 188 & 0 & 0 & 72 & Dirceu Arcoverde & 87 & 1 & 1,2 \\
\hline 17 & Baixa Grande do Ribeiro & 136 & 2 & 1,5 & 73 & Dom Expedito Lopes & 69 & 2 & 2,9 \\
\hline 18 & Barra d'Alcântara & 117 & 1 & 0,9 & 74 & Dom Inocêncio & 287 & 24 & 8,4 \\
\hline 19 & Barras & 776 & 17 & 2,2 & 75 & Domingos Mourão & 104 & 0 & 0 \\
\hline 20 & Barreiras do PI & 81 & 3 & 3,7 & 76 & Elesbão Veloso & 60 & 1 & 1,7 \\
\hline 21 & Barro Duro & 52 & 0 & 0 & 77 & Eliseu Martins & 83 & 0 & 0 \\
\hline 22 & Batalha & 547 & 0 & 0 & 78 & Esperantina & 275 & 1 & 0,4 \\
\hline 23 & Bela Vista do PI & 101 & 3 & 3,0 & 79 & Fartura do PI & 123 & 0 & 0 \\
\hline 24 & Belém do PI & 49 & 0 & 0 & 80 & Flores do PI & 145 & 0 & 0 \\
\hline 25 & Beneditinos & 146 & 0 & 0 & 81 & Floresta do PI & 81 & 7 & 8,6 \\
\hline 26 & Bertolínia & 54 & 1 & 1,9 & 82 & Floriano & 307 & 10 & 3,3 \\
\hline 27 & Betânia do PI & 257 & 1 & 0,4 & 83 & Francinópolis & 36 & 1 & 2,8 \\
\hline 28 & Boa Hora & 133 & 0 & 0 & 84 & Francisco Ayres & 214 & 4 & 1,9 \\
\hline 29 & Bocaina & 43 & 3 & 7,0 & 85 & Francisco Macedo & 50 & 0 & 0 \\
\hline 30 & Bom Jesus & 254 & 3 & 1,2 & 86 & Francisco Santos & 43 & 1 & 2,3 \\
\hline 31 & Bom Princípio do PI & 205 & 1 & 0,5 & 87 & Fronteira & 91 & 0 & 0 \\
\hline 32 & Bonfim do PI & 122 & 0 & 0 & 88 & Geminiano & 134 & 8 & 6,0 \\
\hline 33 & Boqueirão do PI & 155 & 2 & 1,3 & 89 & Gilbués & 248 & 16 & 6,5 \\
\hline 34 & Brasileira & 179 & 2 & 1,1 & 90 & Guadalupe & 36 & 0 & 0 \\
\hline 35 & Brejo do PI & 172 & 11 & 6,4 & 91 & Guaribas & 142 & 1 & 0,7 \\
\hline 36 & Buriti dos Lopes & 508 & 5 & 1,0 & 92 & Hugo Napoleão & 54 & 0 & 0 \\
\hline 37 & Buriti dos Montes & 150 & 3 & 2,0 & 93 & Ilha Grande & 56 & 0 & 0 \\
\hline 38 & Cabeceiras do PI & 290 & 0 & 0 & 94 & Inhuma & 249 & 0 & 0 \\
\hline 39 & Cajazeiras do PI & 74 & 8 & 10,8 & 95 & Ipiranga do PI & 121 & 4 & 3,3 \\
\hline 40 & Cajueiro da Praia & 178 & 2 & 1,1 & 96 & Isaias Coelho & 332 & 27 & 8,1 \\
\hline 41 & Caldeirão Grande do PI & 154 & 0 & 0 & 97 & Itainópolis & 183 & 10 & 5,5 \\
\hline 42 & Campinas do PI & 174 & 20 & 11,5 & 98 & Itaueira & 227 & 4 & 1,8 \\
\hline 43 & Campo Alegre do Fidalgo & 149 & 11 & 7,4 & 99 & Jacobina do PI & 206 & 0 & 0 \\
\hline 44 & Campo Grande do PI & 96 & 3 & 3,1 & 100 & Jaicós & 227 & 1 & 0,4 \\
\hline 45 & Campo Largo do PI & 187 & 1 & 0,5 & 101 & Jardim do Mulato & 89 & 0 & 0 \\
\hline 46 & Campo Maior & 489 & 8 & 1,6 & 102 & Jatobá do PI & 139 & 3 & 2,2 \\
\hline 47 & Canavieira & 115 & 0 & 0 & 103 & Jerumenha & 98 & 2 & 2,0 \\
\hline 48 & Canto do Buriti & 353 & 10 & 2,8 & 104 & João Costa & 124 & 5 & 4,0 \\
\hline 49 & Capitão de Campos & 197 & 0 & 0 & 105 & Joaquim Pires & 391 & 4 & 1,0 \\
\hline 50 & Cap Gervásio de Oliveira & 80 & 9 & 11,5 & 106 & Joca Marques & 0 & 0 & 0 \\
\hline 51 & Caracol & 182 & 0 & 0 & 107 & José de Freitas & 438 & 11 & 2,5 \\
\hline 52 & Caraúbas do PI & 194 & 1 & 0,5 & 108 & Juazeiro do PI & 197 & 2 & 1,0 \\
\hline 53 & Caridade do PI & 55 & 1 & 1,8 & 109 & Júlio Borges & 195 & 0 & 0 \\
\hline 54 & Castelo do PI & 213 & 1 & 0,5 & 110 & Jurema & 123 & 0 & 0 \\
\hline 55 & Caxingó & 102 & 0 & 0 & 111 & Lagoa Alegre & 66 & 0 & 0 \\
\hline 56 & Cocal & 372 & 1 & 0,3 & 112 & Lagoa de São Francisco & 186 & 3 & 1,6 \\
\hline & & & & & 113 & Lagoa do Barro do PI & 183 & 0 & 0 \\
\hline
\end{tabular}


Tabela 3 - Continuação

\begin{tabular}{|c|c|c|c|c|c|c|c|c|c|}
\hline \multirow{2}{*}{$\begin{array}{l}\text { №na } \\
\text { Figura } 1\end{array}$} & \multirow[b]{2}{*}{ Municípios } & \multicolumn{3}{|c|}{ Exames realizados } & \multirow{2}{*}{$\begin{array}{l}\text { №na } \\
\text { Figura } 1\end{array}$} & \multirow[b]{2}{*}{ Municípios } & \multicolumn{3}{|c|}{ Exames realizados } \\
\hline & & $\mathrm{n}^{0}$ & pos & $\%$ pos & & & $\mathrm{n}^{0}$ & pos & $\%$ pos \\
\hline 114 & Lagoa do PI & 86 & 0 & 0 & 169 & Riacho Frio & 181 & 17 & 9,4 \\
\hline 115 & Lagoa do Sítio & 26 & 0 & 0 & 170 & Ribeira do PI & 123 & 4 & 3,3 \\
\hline 116 & Lagoinha do PI & 135 & 0 & 0 & 171 & Ribeiro Gonçalves & 88 & 4 & 4,6 \\
\hline 117 & Landri Sales & 93 & 0 & 0 & 172 & Rio Grande do PI & 145 & 0 & 0 \\
\hline 118 & Luís Correia & 351 & 3 & 0,9 & 173 & Santa Cruz do PI & 57 & 2 & 3,5 \\
\hline 119 & Luzilândia & 122 & 0 & 0 & 174 & Santa Cruz dos Milagres & 57 & 0 & 0 \\
\hline 120 & Madeiro & 188 & 0 & $\begin{array}{r}0 \\
15\end{array}$ & 175 & Santa Filomena & 0 & 0 & 0 \\
\hline $\begin{array}{l}121 \\
122\end{array}$ & $\begin{array}{c}\text { Manoel Emidio } \\
\text { Marcolândia }\end{array}$ & $\begin{array}{r}133 \\
31\end{array}$ & $\begin{array}{l}2 \\
0\end{array}$ & $\begin{array}{r}1,5 \\
0\end{array}$ & 176 & Santa Luz & 103 & 1 & 1,0 \\
\hline $\begin{array}{l}122 \\
123\end{array}$ & $\begin{array}{l}\text { Marcolandia } \\
\text { Marcos Parentes }\end{array}$ & $\begin{array}{l}51 \\
61\end{array}$ & 2 & 3,3 & 177 & Santa Rosa do PI & 77 & 5 & 6,5 \\
\hline 124 & Massapé do PI & 168 & 6 & 3,6 & 178 & Santana do PI & 132 & 5 & 3,8 \\
\hline 125 & Matias Olimpio & 0 & 0 & 0 & 179 & Santo Antonio de Lisboa & 98 & 1 & 1,0 \\
\hline 126 & Miguel Alves & 686 & 0 & 0 & 180 & Sto Antonio dos Milagres & 64 & 0 & 0 \\
\hline 127 & Miguel Leão & 45 & 0 & 0 & 181 & Santo Inácio do PI & 91 & 3 & 3,3 \\
\hline 128 & Milton Brandão & 107 & 0 & 0 & 182 & São Brás do PI & 128 & 1 & 0,8 \\
\hline 129 & Monsenhor Gil & 244 & 1 & 0,4 & 183 & São Felix do PI & 46 & 0 & 0 \\
\hline 130 & Monsenhor Hipólito & 124 & 0 & 0 & 184 & São Francisco de Assis PI & 146 & 2 & 1,4 \\
\hline 131 & Monte Alegre do PI & 202 & 5 & 2,5 & 185 & São Francisco do PI & 235 & 14 & 6,0 \\
\hline 132 & Morro Cabeça no Tempo & 116 & 0 & 0 & 186 & São Gonçalo do Gurguéia & 98 & 10 & 10,2 \\
\hline 133 & Morro do Chapéu do PI & 0 & 0 & 0 & 187 & São Gonçalo do PI & 74 & 1 & 1,4 \\
\hline 134 & Murici dos Portelas & 186 & 5 & 2,7 & 188 & São João da Canabrava & 96 & 0 & 0 \\
\hline 135 & Nazaré do PI & 225 & 4 & 1,8 & 189 & São João da Fronteira & 73 & 0 & 0 \\
\hline 136 & N Senhora de Nazaré & 139 & 0 & 0 & 190 & São João da Serra & 88 & 0 & 0 \\
\hline 137 & N Senhora dos Remédios & 182 & 0 & 0 & 191 & São João da Varjota & 168 & 6 & 3,6 \\
\hline 138 & Nova Santa Rita & 173 & 11 & 6,4 & 192 & São João do Arraial & 0 & 0 & 0 \\
\hline 139 & Novo Oriente do PI & 125 & 1 & 0,8 & 193 & São João do PI & 277 & 32 & 11,6 \\
\hline 140 & Novo Santo Antonio & 98 & 0 & 0 & 194 & São José do Divino & 172 & 0 & 0 \\
\hline 141 & Oeiras & 495 & 16 & 3,2 & 195 & São José do Peixe & 73 & 5 & 6,9 \\
\hline 142 & Olho d'Água do PI & 53 & 0 & 0 & 196 & São José do PI & 236 & 3 & 1,3 \\
\hline 143 & Padre Marcos & 125 & 0 & 0 & 197 & São Julião & 61 & 1 & 1,6 \\
\hline 144 & Paes Landim & 157 & 7 & 4,5 & 198 & São Lourenço do PI & 86 & 1 & 1,2 \\
\hline 145 & Pajeú do PI & 65 & 3 & 4,6 & 199 & São Luis do PI & 137 & 4 & 2,9 \\
\hline 146 & Palmeira do PI & 222 & 3 & 1,4 & 200 & São Miguel da Baixa Gde & 45 & 0 & 0 \\
\hline 147 & Palmeirais & 267 & 0 & 0 & 201 & São Miguel do Fidalgo & 133 & 5 & 3,8 \\
\hline 148 & Paquetá & 126 & 10 & 7,9 & 202 & São Miguel do Tapuio & 126 & 0 & 0 \\
\hline 149 & Parnaguá & 211 & 3 & 1,4 & 203 & São Pedro do PI & 189 & 0 & 0 \\
\hline 150 & Parnaíba & 166 & 1 & 0,6 & 204 & São Raimundo Nonato & 256 & 1 & 0,4 \\
\hline 151 & Passagem Franca do PI & 46 & 0 & $\begin{array}{c}0,0 \\
0\end{array}$ & 205 & Sebastião Barros & 112 & 13 & 11,6 \\
\hline & & & 4 & 24 & 206 & Sebastião Leal & 87 & 0 & 0 \\
\hline 152 & Patos do PI & 164 & 4 & 2,4 & 207 & Sigefredo Pacheco & 167 & 1 & 0,6 \\
\hline 153 & Pau d'Arco & 188 & 0 & 0 & 208 & Simões & 214 & 0 & 0 \\
\hline 154 & Paulistana & 1129 & 3 & 0,3 & 209 & Simplício Mendes & 157 & 6 & 3,8 \\
\hline 155 & Pavussú & 107 & 0 & 0 & 210 & Socorro do PI & 151 & 4 & 2,7 \\
\hline 156 & Pedro II & 611 & 4 & 0,7 & 211 & Sussuapara & 149 & 7 & 4,7 \\
\hline 157 & Pedro Laurentino & 52 & 5 & 9,6 & 212 & Tamboril do PI & 82 & 5 & 6,1 \\
\hline 158 & Picos & 523 & 45 & 8,6 & 213 & Tanque do PI & 56 & 1 & 1,8 \\
\hline 159 & Pimenteiras & 217 & 5 & 2,3 & 214 & Teresina & 335 & 0 & 0 \\
\hline 160 & Pio IX & 139 & 0 & 0 & 215 & União & 720 & 0 & 0 \\
\hline 161 & Piracuruca & 123 & 0 & 0 & 216 & Uruçuí & 114 & 3 & 2,6 \\
\hline 162 & Piripiri & 567 & 1 & 0,2 & 217 & Valença do PI & 186 & 2 & 1,1 \\
\hline 163 & Porto & 134 & 0 & 0 & 218 & Várzea Branca & 105 & 0 & 0 \\
\hline 164 & Porto Alegre do PI & 43 & 0 & 0 & 219 & Várzea Grande & 136 & 0 & 0 \\
\hline 165 & Prata do PI & 32 & 0 & 0 & 220 & Vera Mendes & 46 & 2 & 4,4 \\
\hline 166 & Queimada Nova & 267 & 1 & 0,4 & 221 & Vila Nova do PI & 108 & 0 & 0 \\
\hline 167 & Redenção do Gurguéia & 244 & 9 & 3,7 & 222 & Wall Ferraz & 124 & 10 & 8,1 \\
\hline 168 & Regeneração & 147 & 1 & 0,7 & Total & & 36.399 & 696 & 1,9 \\
\hline
\end{tabular}

Pos $=$ positivos 
Tabela 4 - Distribuição dos municípios, de acordo com a soroprevalência de anticorpos antiT. cruzi em moradores da área rural do Estado do Piauí, no período 1975/2002.

\begin{tabular}{|c|c|c|c|c|c|}
\hline \multirow[t]{2}{*}{ Soroprevalência (\%) } & \multicolumn{2}{|c|}{$\begin{array}{c}\text { Inquérito sorológico } \\
\text { em } 1975 / 1977^{*}\end{array}$} & \multicolumn{2}{|c|}{$\begin{array}{l}\text { Inquérito sorológico } \\
\text { em } 2002\end{array}$} & \multirow{2}{*}{$\begin{array}{c}\text { Análise. } \\
\text { estatística }^{* * *} \\
\mathrm{p}=\end{array}$} \\
\hline & Municípios & $\%$ & Municípios & $\%$ & \\
\hline 0,0 & 11 & 9,7 & 85 & 39,4 & 0,0000000 \\
\hline $0,1-5,0$ & 62 & 54,4 & 105 & 48,6 & 0,318 \\
\hline $5,1-10,0$ & 20 & 17,6 & 20 & 9,2 & 0,028 \\
\hline$\geq 10,1$ & 21 & 18,2 & 6 & 2,8 & 0,0000008 \\
\hline Total & 114 & 100,0 & 216 & 100,0 & \\
\hline
\end{tabular}

* inquérito sorológico nacional, ** qui-quadrado não corrigido

Tabela 5 - Soropositividade para anticorpos antiT. cruzi em moradores da área rural do Estado do Piauí, 2002, de acordo com a faixa etária e sexo.

\begin{tabular}{|c|c|c|c|c|c|c|c|c|c|}
\hline \multirow{2}{*}{$\begin{array}{l}\text { Faixa } \\
\text { etária }\end{array}$} & \multicolumn{3}{|c|}{ Mulheres } & \multicolumn{3}{|c|}{ Homens } & \multicolumn{3}{|c|}{ Total } \\
\hline & exam & $\mathrm{sp}$ & $\mathrm{sp}(\%)$ & exam & $\mathrm{sp}$ & sp (\%) & exam & $\mathrm{sp}$ & $\mathrm{sp}(\%)$ \\
\hline $00-04$ & 1.894 & 1 & 0,1 & 1.994 & 0 & $\cdots$ & 3.888 & 1 & 0,1 \\
\hline 05-09 & 2.234 & 4 & 0,2 & 2.306 & 5 & 0,2 & 4.540 & 9 & 0,2 \\
\hline $10-14$ & 2.132 & 6 & 0,3 & 2.258 & 6 & 0,3 & 4.390 & 12 & 0,3 \\
\hline $15-19$ & 1.960 & 5 & 0,3 & 1.919 & 4 & 0,2 & 3.879 & 9 & 0,2 \\
\hline 20-29 & 3.052 & 22 & 0,7 & 2.419 & 13 & 0,5 & 5.471 & 35 & 0,6 \\
\hline 30-39 & 2.324 & 31 & 1,3 & 1.831 & 25 & 1,4 & 4.155 & 56 & 1,4 \\
\hline 40-49 & 1.844 & 85 & 4,6 & 1.448 & 37 & 2,6 & 3.292 & 122 & 3,7 \\
\hline $50-59$ & 1.619 & 102 & 6,3 & 1.235 & 86 & 7,0 & 2.854 & 188 & 6,6 \\
\hline $60-69$ & 1.024 & 72 & 7,0 & 1.021 & 60 & 5,9 & 2.045 & 132 & 6,5 \\
\hline 70-79 & 598 & 45 & 7,5 & 560 & 39 & 7,0 & 1.158 & 84 & 7,3 \\
\hline$>=80^{*}$ & 366 & 28 & 7,7 & 361 & 20 & 5,5 & 727 & 48 & 6,6 \\
\hline otal & 19.047 & 401 & 2,1 & 17.352 & 295 & 1,7 & 36.399 & 696 & 1,9 \\
\hline
\end{tabular}

* incluindo com idade ignorada, exam = total de examinados; $\mathrm{sp}=$ soropositivos pelo teste de imunofluorescência indireta em eluato de sangue

Comparando-se a distribuição, por faixa etária, das pessoas soropositivas do presente inquérito com a distribuição das pessoas soropositivas do inquérito sorológico nacional, observa-se significativa redução do número de pessoas com idades abaixo de 30 anos, no intervalo de 25 anos, 1977/2002 (Figura 2). Isto revela que o controle da transmissão vetorial intradomiciliar determina de imediato a queda na prevalência da infecção nas populações de crianças e jovens, transformando-as em sensores da eficácia das medidas de controle.

No grupo de 27.971 pessoas examinadas com $\geq 10$ ou mais anos de idade foram identificadas $686(2,5 \%)$ positivas para anticorpos antiT. cruzi, sendo, $407(4,1 \%)$ entre as 9.850 analfabetas e $279(1,5 \%)$ entre as 18.121 alfabetizadas $\left(\chi^{2}\right.$ $=179,25 ; \mathrm{p}=0,0000000 ; \mathrm{gl}=1 ; \mathrm{RR}=2,68[2,31<\mathrm{RR}$ $<3,12]$ ), mantendo-se a associação inversa com 0 grau de instrução (Tabela 6), mesmo considerando pares do mesmo sexo e faixa etária.

No total de 36.399 pessoas examinadas, $982(2,7 \%)$, com idade média de 56 anos, referiram recepção de sangue. A presença de anticorpos antiT. cruzi foi registrada em 32 (3,3\%) pessoas das 982 que receberam transfusão de sangue e em $664(1,9 \%)$ das 35.417 que não receberam transfusão $\left(\chi^{2}=9,76 ; \mathrm{p}=0,0017 ; \mathrm{gl}=1 ; \mathrm{RR}=1,74[1,23<\mathrm{RR}<2,46]\right)$.

No grupo de 12.426 mulheres examinadas com $\geq 15$ anos de idade, $2.267(18,2 \%)$ afirmaram ter apresentado

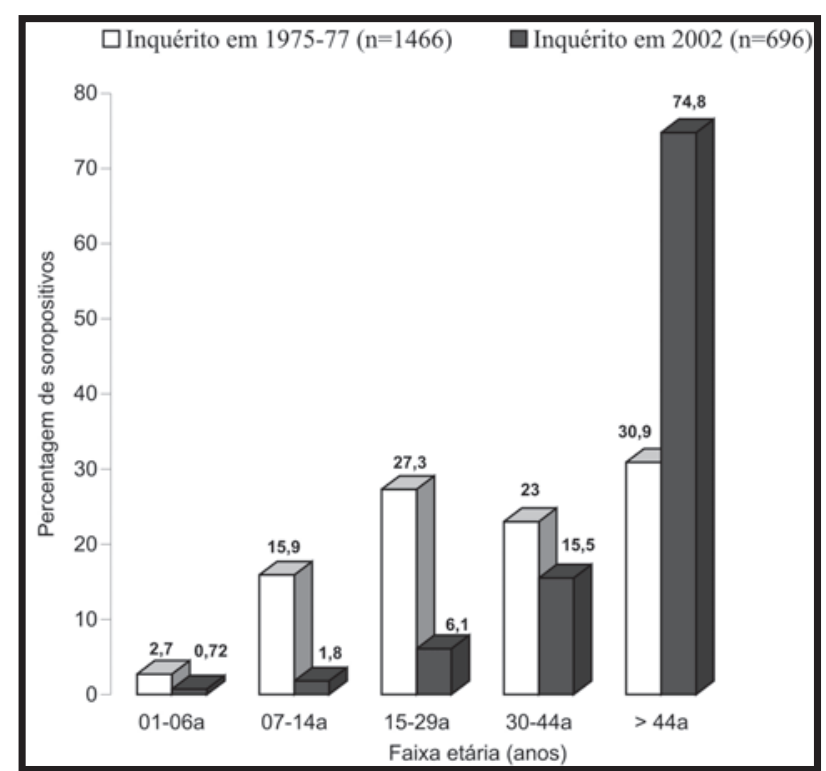

Figura 2 - Evolução da soropositividade para anticorpos antíT.cruzi em moradores da área rural do Estado do Piauí, 1975/2002, conforme a faixa etária.

Tabela 6 - Soropositividade para anticorpos antiT. cruzi em moradores da área rural com idade $\geq 10$ anos do Estado do Piauí, 2002, de acordo com o grau de escolaridade.

\begin{tabular}{lccc}
\hline Escolaridade & \multicolumn{3}{c}{ Moradores da área rural com idade $\geq 10$ anos } \\
\cline { 2 - 4 } & examinados & soropositivos & Soropositivos (\%) \\
\hline Analfabetos & 9.850 & 407 & 4,1 \\
Primeiro grau & 17.095 & 268 & 1,6 \\
Segundo grau & 919 & 10 & 1,1 \\
Terceiro grau & 107 & 1 & 0,9 \\
\hline Total & 27.971 & 686 & 2,5 \\
\hline
\end{tabular}

abortamento espontâneo. A presença de anticorpos antiT. cruzi foi registrada em $123(5,4 \%)$ das 2.267 mulheres que referiram abortos e em 267 (2,6\%) das 10.159 mulheres que não referiram abortos $\left(\chi^{2}=47,71 ; \mathrm{p}=0,0000000 ; \mathrm{gl}=1\right.$; $\mathrm{RR}=2,06[1,68<\mathrm{RR}<2,54])$.

\section{DISCUSSÃo}

$\mathrm{Na}$ análise crítica do trabalho, consideramos boa a qualidade das informações e material obtidos, tendo em vista que a maioria do pessoal empregado na coleta tinha experiência de inquéritos anteriores patrocinados pela SUCAM e Fundação Nacional de Saúde. 0 desempenho da pesquisa pode ser considerado bom, seis municípios não realizaram os inquéritos, em função da insuficiência de pessoal na ocasião destinada ao trabalho, enquanto a população estudada permitiu estimar a prevalência da infecção chagásica em cada município. A não participação de cerca de 33\% da população estimada deveu-se à realização de uma única visita aos domicílios, sem comprometer a representatividade (Tabela 1). A decisão de uma visita aos domicílios foi de natureza econômica, outras visitas implicariam em mais gastos com viagens, diárias e combustível. 
A ocorrência de casos de leishmanioses, principalmente nas áreas das Regionais de Saúde de Parnaíba, Barras, Piripiri, Teresina e Campo Maior (norte do estado), pode determinar reações cruzadas no teste de IFI utilizado na pesquisa, superestimando os valores das prevalências para essas áreas, embora, a queda registrada entre o inquérito sorológico nacional de 1975 e o inquérito atual, indique o contrário. Do mesmo modo que os autores do inquérito sorológico nacional ${ }^{7}$, consideramos a boa qualidade do kit e a grande experiência dos técnicos como fatores determinantes do menor índice de resultados duvidosos. A influência da reação cruzada na prevalência da infecção chagásica no Estado do Piauí está sendo avaliada na pesquisa em desenvolvimento para a confirmação sorológica com testes de ELISA e de hemaglutinação em soros.

Esse inquérito sorológico foi realizado concomitantemente com o inquérito triatomínico nas unidades domiciliares sorteadas. Ambos foram realizados por decisão do governo estadual, atendendo as solicitações dos governos municipais acerca de informações atuais sobre da doença de Chagas, de tal modo que lhes permitissem avaliar a eficácia das medidas de controle e a criação de estratégias para o adequado atendimento dos pacientes com cardiopatia e/ou megas digestivo. A Secretaria de Saúde do Estado (SESAPI) participou de todas as etapas da pesquisa, viabilizando o deslocamento e disponibilizando todo o material necessário para o treinamento de técnicos, coleta de sangue e captura de triatomíneos, enquanto as secretarias municipais participaram fornecendo pessoal para a coleta de sangue e captura de triatomíneos. Como produto da realização concomitante dos dois inquéritos verificou-se a estreita associação entre a soroprevalência e a presença de triatomíneos, ao se constatar que 64\% da área com pessoas soropositivas apresentaram captura positiva para T. brasiliensis e T. pseudomaculata. Isto adquire importância na avaliação do impacto do controle da transmissão vetorial sobre os índices de soroprevalências. Neste contexto, destacamos que a queda na soroprevalência foi maior nos municípios que tinham T. infestans em 1975, do que nos municípios que não tinham. Outro dado observado no inquérito entomológico foi de que a eliminação do $T$. infestans desses municípios associou-se com a eliminação do P. megistus, T. brasiliensis e T. pseudomaculata, indicando-nos que as medidas destinadas ao controle de vetores intradomiciliares podem ter como impacto a eliminação de vetores peridomiciliares.

Todas as amostras de sangue coletadas e os triatomíneos capturados foram encaminhados para o Departamento de Microbiologia e Parasitologia da Universidade Federal do Piauí; conferidas as identificações eram distribuídos para os laboratórios de sorologia e entomologia. Não houve perda de amostras de sangue, todas foram processadas e submetidas ao teste sorológico de IFI.

A redução na transmissão vetorial no Estado do Piauí, no período 1975/2002, pode ser observada e dimensionada através da comparação dos atuais resultados com os obtidos nos inquéritos sorológico e entomológico nacionais ${ }^{15} 16$. Observa-se significativa redução da área de ocupação de pessoas positivas para anticorpos antiT. cruzi de $90,4 \%$ para
$60,6 \%$, queda na soroprevalência de $4 \%$ para $1,9 \%$ com taxas inferiores a $1 \%$ entre as pessoas com menos de 30 anos de idade, eliminação do Triatoma infestans e diminuição da densidade e domiciliação do T. brasiliensis ${ }^{7}$. Outro indicador indireto da redução da transmissão vetorial a ser considerado é o pequeno número de domicílios com mais de um familiar soropositivo no presente trabalho. Esse indicador deveria ser utilizado sistematicamente nas avaliações do processo de transmissão, por traduzir a força ou atividade da transmissão vetorial de uma área, dependente da variação do número de domicílios com mais de um familiar soropositivo. Esses dados configuram a eficácia das medidas de controle vetorial instituídas no Estado do Piauí.

Por outro lado, a ausência na literatura de dados sobre a soroprevalência entre candidatos a doação de sangue ou mesmo do potencial de transmissão por transfusão de sangue no Piauí, não nos permitem uma avaliação longitudinal desse mecanismo de transmissão. A maior soropositividade entre as pessoas que receberam transfusão de sangue observada no inquérito atual, como resultado geral, indica transtornos no controle da via transfusional no Estado do Piauí, os quais deverão ser abordados em estudo específico a ser realizado posteriormente.

Com a possível eliminação da transmissão vetorial intradomiciliar e redução da transfusional, o foco de atenção migra para os mecanismos de transmissão congênita, acidental, oral ou por transplante de órgãos. A incidência da transmissão congênita da doença de Chagas pode variar em função de áreas, populações estudadas e modelos de estudos. A falta de padronização nas investigações tem dificultado as análises sobre sua incidência, produzindo dimensionamento irreal do problema ${ }^{4}$.

No presente inquérito, ficou demonstrada a participação do componente etiológico chagásico na prevalência de abortamento espontâneo no Estado do Piauí, principalmente nas áreas com maiores prevalências da infecção chagásica. Esse resultado conduz à hipótese de que quanto maior o nível de aborto espontâneo menor a chance de identificação de crianças infectadas pelo $T$. cruzi em inquéritos sorológicos em áreas com transmissão vetorial controlada ou inativa, a despeito das possíveis diferenças regionais. Como indicação dessas diferenças regionais, investigadas em inquérito sorológico nacional em menores de 5 anos, estudos realizados em Cássia dos Coqueiros-São Paulo ${ }^{19}$, São Felipe-Bahia ${ }^{2}$ não encontraram diferença significativa nas prevalências de abortamento espontâneo entre mulheres soropositivas e soronegativas para a doença de Chagas. Rassi cols ${ }^{15}$ em estudo retrospectivo envolvendo 145 mulheres chagásicas, predominantemente de Goiás e Minas Gerais, revelaram história de aborto espontâneo em $21(14,5 \%)$ e de transmissão maternal do Trypanosoma cruzi em 2 (1,4\%) portadoras de cardiopatia crônica.

A história de aborto espontâneo foi registrada em 123 (31,5\%) das 390 mulheres soropositivas e em 2.144 (17,8\%) das 12.036 mulheres soronegativas, com $\geq 15$ anos de idade, estabelecendo um risco de aborto espontâneo 1,8 vezes maior $(\mathrm{p}<0,05)$ para as mulheres com anticorpos antiT cruzi. 
Isto não define a presença de transmissão congênita da doença de Chagas, mas aponta para sua possibilidade que será explorada com maior acuidade em investigação posterior na qual avaliaremos a história obstétrica de mulheres chagásicas pareadas com controles não chagásicas do Estado do Piauí, abordando aspectos fundamentais como a nutrição, o tabagismo e o alcoolismo. Considerando-se o número de casos em menores de cinco anos encontrado no presente estudo como indicador de transmissão congênita da doença de Chagas no Estado Piauí (Tabela 5), admitimos baixa a probabilidade de sua ocorrência.

Em áreas rurais nas quais a transmissão vetorial ocorre ou ocorreu no intradomicílio têm sido identificada maior prevalência da infecção chagásica no sexo feminino $0^{5611}, 0$ que tem sido atribuída ao maior tempo de permanência das mulheres no interior dos domicílios ou ao hábito de dormirem mais próximas das paredes do que os homens. Essas questões precisam ser definidas por investigações bem conduzidas, pois estão abertas as interrogações sobre gênero e susceptibilidade a infecção pelo $T$. cruzi. No presente inquérito, de acordo com os resultados obtidos em diversos estudos pontuais realizados no Piauí ${ }^{3} 9^{10}$, evidenciou-se maior positividade sorológica entre as mulheres, indicando que a transmissão vetorial ocorreu principalmente no interior das casas, apesar dos principais triatomíneos do estado (Triatoma brasiliensis e Triatoma pseudomaculata) serem mais freqüentemente capturados no peridomicílio.

0 perfil da curva da prevalência em função da faixa etária mostra o deslocamento dos maiores valores para a direita, configurando a correlação direta entre a incidência da infecção e o tempo de vida e a interrupção da transmissão em áreas nas quais o principal vetor é intradomiciliar como o T. infestans. Curvas com esse desenho foram observadas nos estudos realizados em áreas como Pains, Iguatama ${ }^{11}$, Berilo $^{1}$ e Virgem da Lapa ${ }^{5}$, Minas Gerais, todas com transmissão vetorial interrompida há pelo menos 20 anos.

A associação da doença de Chagas com as péssimas condições sociais tem sido referida em todos os estudos epidemiológicos realizados em áreas rurais da América Latina. 0 analfabetismo tem se caracterizado como o principal marcador social da infecção chagásica. No presente estudo, a soroprevalência entre os analfabetos foi duas vezes maior do que na população geral e 4,5 vezes maior do que no grupo de pessoas com terceiro grau de escolaridade. Esses dados configuram a história do modelo de desenvolvimento instalado no Brasil colônia: à maioria, não a educação, não a propriedade da terra e a uma minoria dominadora, educação escravocrata e latifúndios impróprios.

Considerando os limites desse trabalho podemos observar que a infecção chagásica humana no Estado do Piauí está em queda, como consequiência da eficácia no controle da transmissão vetorial, apesar da existência de alguns municípios com elevadas taxas de infecção, sem, no entanto, representar risco significativo para instalação ou manutenção do ciclo doméstico.

Como produto desse inquérito estão sendo coletadas amostras de sangue venoso das pessoas soropositivas e pares soronegativas para confirmação sorológica através de testes de ELISA e hemaglutinação, com prioridade para os menores de 20 anos, diante da possibilidade de serem submetidos ao tratamento etiológico. Outro produto será a realização de uma investigação para dimensionar a influência da infecção chagásica e da morbidade na fertilidade e nas intercorrências da gravidez.

\section{AGRADECIMENTOS}

Os autores agradecem aos agentes de saúde, guardas sanitários, enfermeiras, técnicos, auxiliares administrativos das secretarias de saúde dos municípios e do Estado do Piauí pela adesão, participação e realização desse trabalho. Aos responsáveis pelo Laboratório de Reativos Diagnósticos de BioManguinhos pelo fornecimento dos testes IFI-Chagas utilizados na identificação da infecção chagásica. Aos técnicos José de Souza Nogueira, José Henrique Furtado e Júlio César Miguel pela colaboração na realização dos testes sorológicos. A Heloisa Maria Nogueira Diniz pela composição da Figura 1.

\section{REFERÊNCIAS BIBLIOGRÁFICAS}

1. Araújo RM. Morbidade da doença de Chagas no município de Berilo, Minas Gerais. Estudos seccional e evolutivo de 10 anos (1987-1997). Tese de doutorado. Instituto Oswaldo Cruz, Fundação Oswaldo Cruz, Rio de Janeiro, RJ, 1998.

2. Batista C, Teixeira G, Macedo V. Prevalência de abortamento e prematuridade em uma área endêmica para a doença de Chagas. In: Anais do XVI Congresso da Sociedade Brasileira de Medicina Tropical, Natal, p.50. 1980 .

3. Bento DNC, Freitas M, Pinto AS. Epidemiologia da doença de Chagas nos municípios de Castelo do Piauí e Pedro II, Estado do Piauí, Brasil. Revista da Sociedade Brasileira de Medicina Tropical 22:73-79, 1989.

4. Bittencourt AL. Transmissão congênita. In: Cançado JR (ed) Doença de Chagas, $1^{\underline{a}}$ edição, Belo Horizonte, Minas Gerais, p.100-129, 1968.

5. Borges-Pereira J. Doença de Chagas humana: Estudo da infecção crônica, morbidade e mortalidade em Virgem da Lapa, MG, Brasil (1976-1996). Tese de doutorado, Instituto Oswaldo Cruz, Fundação Oswaldo Cruz, Rio de Janeiro. 1997.

6. Borges-Pereira J, Castro JAF, Campos JHF, Nogueira JS, Zauza PL, Marques P, Cardoso MA, Britto C, Araújo AJG. Estudo da infecção e morbidade da doença de Chagas no município de João Costa - Parque Nacional Serra da Capivara, Piauí, Brasil. Revista da Sociedade Brasileira de Medicina Tropical 35: 315-322, 2002.

7. Camargo ME, Silva JR, Castilho EA, Silveira AC. Inquérito sorológico da prevalência da infecção chagásica no Brasil, 1975-1980. Revista do Instituto de Medicina Tropical de São Paulo 26: 192-204, 1984.

8. Castro JAF, Borges-Pereira J, Gonçalves MER, Pereira FCA, Silva AR, Costa MGP, Cardoso MBS, Leal MJB, Castro AE, Salmito MA, Pereira LRM, CorreiaLima FG, Coura JR. Doença de Chagas no estado do Piauí, Brasil, 2002: I - resultados preliminares do inquérito triatomínico. Revista da Sociedade Brasileira de Medicina Tropical 36 (supl I) : 376, 2003.

9. Correia-Lima FG. Doença de Chagas no município de Oeiras, Piauí - estudo seccional nas localidades de Colônia e Oitis. Dissertação de Mestrado, Faculdade de Medicina da Universidade Federal do Rio de Janeiro, RJ, 1976.

10. Correia-Lima FG, Figueiredo PZ, Portella-Nunes JN. Prevalência da infecção chagásica na população humana determinada pelo teste de imunofluorescência indireta em 24 municípios do Piauí. Revista da Sociedade Brasileira de Medicina Tropical 10: 17-25, 1976.

11. Coura JR, Abreu LL, Dubois LEG, Correia-Lima FG, Arruda Jr ER, Willcox HPF, Anunziato N, Petana W. Morbidade da doença de Chagas. II - Estudos 
seccionais em quatro áreas de campo no Brasil. Memórias do Instituto Oswaldo Cruz 79: 101-124, 1984.

12. Figueiredo PZ, Correia-Lima FG, Portella-Nunes JN. Doença de Chagas: primeiros casos autóctones no estado do Piauí. Revista da Sociedade Brasileira de Medicina Tropical 9: 105-107, 1975.

13. Instituto Brasileiro de Geografia e Estatística (IBGE). http:// www.ibge.gov.br/censo 2000 .

14. Neiva A, Penna B. Viagem científica pelo norte da Bahia, sudoeste de Pernambuco, sul do Piauí e de norte a sul de Goiás. Memórias do Instituto Oswaldo Cruz 8: 74-224, 1916.

15. Rassi A, Amato Neto V, Rassi GG, Amato VS, Rassi Junior A, Luquetti AO, Rassi SG. Busca retrospectiva da transmissão maternal da infecção chagásica em pacientes na fase crônica. Revista da Sociedade Brasileira de Medicina Tropical 37: 485-489, 2004.
16. Silveira AC, Feitosa VR, Borges R. Distribuição de triatomíneos capturados no ambiente domiciliar, no período 1975/83, Brasil. Revista Brasileira de Malariologia e Doenças Tropicais 36:15-312, 1984.

17. Silveira AC, Vinhaes MC. Doença de Chagas: aspectos epidemiológicos e de controle. Revista da Sociedade Brasileira de Medicina Tropical 31 (supl II): 15-60, 1998

18. Souza SL, Camargo ME. The use of filter paper blood smear in a practica fluorescent test for American Trypanosomiasis serodiagnosis. Revista do Instituto de Medicina Tropical de São Paulo 8: 255-258, 1966.

19. Teruel JR, Nogueira JL. Perdas fetais em área de alta prevalência de moléstia de Chagas crônica. Revista do Instituto de Medicina Tropical de São Paulo 12:239-244, 1970 